\title{
Finite size effects in Neutron Star and Nuclear matter simulations
}

\author{
P. A. Giménez Molinellia ${ }^{\mathrm{a}}$ C. O. Dorso ${ }^{\mathrm{a}}$ \\ ${ }^{a}$ Departamento de Fsica, Facultad de Ciencias Exactas y Naturales, Universidad de Buenos Aires and IFIBA, \\ CONICET, Cuidad Universitaria, Buenos Aires 1428, Argentina
}

\begin{abstract}
In this work we study molecular dynamics simulations of symmetric nuclear matter using a semi-classical nucleon interaction model. We show that, at sub-saturation densities and low temperatures, the solutions are non-homogeneous structures reminiscent of the "nuclear pasta" phases expected in Neutron Star Matter simulations, but shaped by artificial aspects of the simulations. We explore different geometries for the periodic boundary conditions imposed on the simulation cell: cube, hexagonal prism and truncated octahedron. We find that different cells may yield different solutions for the same physical conditions (i.e. density and temperature). The particular shape of the solution at a given density can be predicted analitically by energy minimization. We also show that even if this behavior is due to finite size effects, it does not mean that it vanishes for very large systems and it actually is independent of the system size: The system size sets the only characteristic length scale for the inhomogeneities.

We then include a screened Coulomb interaction, as a model of Neutron Star Matter, and perform simulations in the three cell geometries. In this case, the competition between competing interactions of different range produces the well known nuclear pasta, with (in most cases) several structures per cell. However, we find that the results are affected by finite size in different ways depending on the geometry of the cell. In particular, at the same physical conditions and system size, the hexagonal prism yields a single structure per cell while the cubic and truncated octahedron show consistent results with more than one structure per cell. In this case, the results in every cell are expected to converge for systems much larger than the characteristic length scale that arises from the competing interactions.
\end{abstract}

Keywords: Nuclear Astrophysics, Nuclear Matter phase transition, Molecular Dynamics simulations, Finite Size Effects

\section{Introduction}

In the inner crust of Neutron Stars, nucleons (protons and neutrons) exist at low temperatures and densities embedded in a (charge neutralizing) degenerate electron gas. Under those conditions, instead of forming the usual quasi-spherical nuclei found in Earth ("normal" nuclei), nucleons behave like a complex fluid called Neutron Star Matter (NSM). In NSM, nucleons attract each other through the short-ranged Nuclear Interaction while protons, in addition, repel each other through the Coulomb interaction screened by the electron gas. Studies of low density

Email address: pagm@df .uba.ar (P. A. Giménez Molinelli)

Preprint submitted to Nuclear Physics A

July 3, 2021 
NSM have found that the attractive-repulsive interplay of nuclear and Coulomb forces may drive nucleons to take non-uniform configurations which are collectively known as "nuclear pasta" [1].

\subsection{Non-homogeneous phases of Neutron Star Matter}

In the context of nuclear systems, the existence of nucleer pasta was early proposed in a pioneering work by Ravenhall et.al.[1]. There the authors argued that while at low densities the interplay between nuclei surface and Coulomb self-energies alone produce the almost spherical normal nuclei, that is not the case at higher densities.

When the volume fraction of the dense matter phase ranges from $0.1 \rightarrow 1$, the contribution to the Coulomb energy coming from neighboring nuclei would rival in importance the Coulomb self-energy of a given nuclei

To analyze the effect of the long range Coulomb interaction at sub-saturation densities they used a static compressible liquid drop model in the Wigner-Seitz approximation. The WignerSeitz approximation was devised to simplify calculations in a charge neutral lattice of arbitrary shape. It consists in replacing the charge neutral unit cell with another cell of a simplified geometry, adecuate for the chosen lattice dimensionality: a sphere in $3 D$, a disc or cilinder in $2 D$ or a slab in $1 D$. Lattice Coulomb energy is included implicitly by making the electrostatic potential vanish at the cell's boundary.

The calculations from [1] were made at zero temperature with a proton fraction $x=0.3$. Nucleons were assumed to be arranged in dense matter regions at saturation density $\rho_{0}$ but filling only a fraction of space. ith this model they found that for a rather wide range of densities, NSM arranged in these idealized lattice geometries is more stable than uniform matter or normal nuclei. As described by the authors, these non uniform phases range in order of increasing density from spherical (3D) "nuclei", cilindrical (2D) "nuclei", passing through a slab-like "nuclei", then to cilindrical "bubbles", then spherical "bubbles", until finally at $\rho \approx 0.85 \rho_{0}$ the system becomes uniform

For each geometry the unit cell has a characteristic length $r_{c}$ which is the radius for $3 D$ and $2 D$, and the half width of the $1 D$ lattice. Although the value of this parameter is not given, the fact that the slabs are stable at volume fraction 0.5 , where their width is of order $10 \mathrm{fm}$ suggests that the same is true for $r_{c}$.

While groundbreaking, this work assumed possible shapes a priori by restricting them to some simple idealized geometries. The authors make no mention to a possible dependence of the results on the unit cell's size $r_{c}$. But since surface effects are supposed to be at the core of the whole phenomenon, such drawback might be critical.

Later on, to address this criticisms, Williams and Koonin improved the calculations[2] by allowing NSM to assume any arbitrary configuration within a cubic unit cell of side $L$ (which is not to be mistaken with a Wigner-Seitz cell) under periodic boundary conditions (PBC). To allow for arbitrary configurations they divided the unit cell into a cubic lattice within that cell. The local number density of isospin symmetric NSM at each site was relaxed to minimize an energy functional. he energy functional was divided explicitly and a priori in bulk, surface and Coulomb terms. For the proton-proton Coulomb interaction, the zero temperature Thomas-Fermi approximation was employed [3], under which the Coulomb potential is screened and takes the form:

$$
V_{T F}(r)=\mathrm{q}^{2} \frac{e^{-r / \lambda}}{r}
$$


Since the Thomas-Fermi screening length is much larger than any other length scale involved, the authors argued that it is a good approximation to include electrons as a uniform background of negative charge, just to neutralize the system. The minimization of energy was carried out through a careful and complex procedure which involved variation of the local number density, the overall mean density and the unit cell's size and. Due to computational limitations, the largest cell size considered was of $L=32 \mathrm{fm}$. Also, the authors state that the choice of a cubic cell was made solely for computational convenience but other geometries would be valid. They are aware that this poses a bias on the possible density configurations they may obtain, but also state that their calculations contemplate all possible density configurations which have periodic cubic symmetry.

With this model their results inculde, in addition to the original "pasta", a new shape (dubbed "cross") which they describe as a slab with regular holes. According to the authors, this new shape is an energy minimum only for a very small range of densities, and so shallow that it "would be washed out by finite temperature"(sic). Most interestingly, by increasing the mean density adiabatically this phase transforms into a regular slab, but in a larger cell. Although the authors do not mention it, this is explicit proof that at least within this model, the shape that minimizes the energy depends on the arbitrary cell size, and that in order to find the true minimum energy configuration at a given density, several system sizes must be explored to avoid (or at least exhibit) finite size effects.

More recently, Monte Carlo and Molecular Dynamics simulations have become popular tools to study the behavior of NSM at sub-saturation densities ([4, 6, 8]. These simulations usually consider $(n, p, e)$ matter (neutrons, protons and electrons only) at a fixed number of particles, volume and temperature. Electrons, however, are never included explicitly in the simulations but are treated either as an ideal Fermi gas or a uniform background charge distribution for the double purpose of achieving charge neutrality and screening the Coulomb interaction between protons.

The advantage of using a, for want of a better word, "microscopic" and dynamical approach is that nucleons are treated individually instead of dealing with local densities. And instead of artificially splitting the energy contributions a priori in bulk, surface and Coulomb terms, pairwise interaction potentials are used. The dynamics itself then arranges the nucleons in whatever shape is optimal for the model without any bias, except for the geometry and size of the simulation cell.

\subsection{Other systems with non-uniform phases}

Similar non-homogeneous structures are found in solvated di-block copolymers [18] and are, actually, inherent to any system with competing interacions of different ranges (see [20, 21] and references therein). Phenomenologically, this usually manifests as a competition between bulk and surface or interfacial energies which is settled by adopting a geometry such that its surface is minimal [22], while subject to constraints or frustration.

The formation of nuclear pasta has even been described as a frustration of surface minimization produced by Coulomb interaction[20]. In any case, even partial or frustrated, surface minimization seems to lie at the core of the nuclear pasta phenomenon.

In mathematics the problem of finding the minimal surface subject to certain boundary conditions, is known as the "Plateau" problem[23]. A minimal surface can be defined as a surface with zero average curvature at every point. All the usual pasta, plus Williams and Koonin's Crosses, CMD's “jungle-gym”[8] (known as the plumber's nightmare in polymer physics), as well as the Gyroid and Double Diamond structures [17] are solutions with cubic symmetry to this well studied problem [25]. For example, HF models for NSM [16, 24] yield the so called Schwarz's 
P-Surface and D-Surface [25]. CMD's jungle-gym also resembles the Schwarz's P-Surface. Watanabe et. al. report that with QMD they find several "triply periodic" structures [19].

It seems that almost every pasta-like structures found in NSM simulations are actually triply periodic minimal surfaces of cubic symmetry, and as such, may be constrainted to some extent by the geometry and symmetries of the (cubic) primitive cell, not only by its size. Most notably, whenever a model yields only one structure per cell [24].

And since the "triply periodic" nature of most nuclear structures might be related to the symmetries of the cubic cell, exploring cell geometries with different symmetries might be enlightening.

\subsection{Simulations and finite size considerations}

In any kind of particle-based simulations finite size is always a concern, but it is assumed that for large enough cells, the so-called "finite size effects" would become negligible. That, however, is not always true. At least not for every observable. In [9, 11, 12], for example, grand canonical montecarlo simulations are performed for a Lennard-Jones fluid at densities that correspond to phase coexistence in the (mean field) Van der Waals approximation. The liquid phase in these simulations appears ordered in non-homogeneous structures eerily reminiscent to those observed in systems with competitive interactions. We shall refer to those structures as "pseudo-pasta" to distinguish them from "true" pasta, arising in systems with competing interactions.

In [12], the authors show both analitically and numerically that at the liquid-vapor coexistence region of a Lennard-Jones fluid, the liquid phase is shaped in a very distinct way for a given density and temperature. They assume a cubic cell under Periodic Boundary Conditions (PBC) and show that if that cell is large enough, the shapes of these pseudo-pasta are limited to one spherical drop, one cilindrical rod, one slab, one cilindrical hole or one spherical hole in the simulation cell (in order of increasing number density). Small transitional density regions exist due to interfacial effects related to the finite range of the interactions, but these effects become smaller as system size is increased and/or temperature is lowered. In the limit of infinitely large and/or cold systems $(L \rightarrow \infty)$ the transition densities can be calculated exclusively from surface minimization.

This behavior is observed for systems of various sizes and, based on scaling properties of the Landau free energy, it is shown that in the $L \rightarrow \infty$ the size dependence of every intensive quantity vanishes. This is so because their size dependence appears explicitly through surface to volume ratios which become (slowly) negligible as the size is increased. But most notably, it is also shown for any large enough system, the transition from uniform liquid to uniform vapor is made in steps, passing through the same sequence of shapes expected in NSM (spherical bubble, cilindrical bubble, slab, rod and spherical droplet) but only one per simulation cell.

Unaware of most of these works, in [10] we found the same structures for simulations of both Lennard-Jones and symmetric NM for several semi-classical interaction models for the nuclear interaction. Our simulations were done at constant volume and almost zero temperature and without Coulomb interaction. We explained these pseudo-pasta structures as minimal surface configurations under cubic PBC, and shown them to be the most stable configuration independently of system size (consistently with the results from [12]). Thus, we concluded that these behavior is intrinsic to the finite size of simulations under PBC, independently of the system size. That is to say,"finite size" effects are not due to the system being small, as is usually interpreted. This scenario is very often ignored, but is inherent to simulations of any LJ-like system.

Given this background, it is feasible that at least some of the pasta structures found in NSM simulations (with Coulomb interaction) might be biased by finite size and the particular boundary 
conditions imposed and should be explored. It is even possible that some may be entirely an artifact of the simulation itself, specially if the size of the simulation cell is small and for models that produce only one estructure per cell[16].

And in any case, ignoring this fact may lead to wrong estimates of the true scale of the density fluctuations, which in the case of nuclear pasta is of paramount importance[6].

\subsection{Aim and organization of this work}

In this work we aim to show that in molecular dynamics simulations of ideal symmetric nucelar matter under periodic boundary conditions, the spatial configuration of the solution at subsaturation densities and low temperatures is determined exclusively by the artificial periodicity of the cell. The same is true for a large variety of systems which we shall call Lennard-Jones-like (LJ-like) systems. Those are systems interacting through potentials that are repulsive at very short ranges and attractive at short-ranges. For this very general class of systems, the binding energy energy can be divided into a volume plus a surface (or interface) term:

$$
E=a_{V} V+a_{S} S
$$

where $a_{V}$ and $a_{S}$ may be functions of the temperature, density or other variables. In the case of nuclear systems, this is nothing more than a truncation of the semi-empirical mass formula. As we argued in[10], energy minimization at fixed number density is achieved by surface minimization.

In section 3 we explore several possible symmetries for the simulation cell. We argue that even though cubic PBC are the most usual choice for numerical simulations due to their simplicity and the low cost of their computational implementation, they're not by any measure the only option available. In particular, we explore cubic, hexagonal-prism and truncated octahedron

Based on the arguments exposed above, in section 3.2 we search for the minimum surface configuration as a function of the volume fraction of the cell which is occupied. We do so in the three cell geometries described in section 3 and show that the configuration predicted for a given volume fraction depends on the arbitrary choice of $\mathrm{PBC}$.

The predictions of section 3.2 are verified with molecular dynamics simulations of symmetric NM within the framework of the semi-classical model CMD, which is described in section 2 Simulations were done at almost zero temperature, at three relevant densities, for two system sizes and in the three different cell geometries. The results of the simulations themselves are presented and discussed in section 4 .

In addition, and since the motivation for this research is the simulation of NSM which includes competing interactions, we also performed simulations with the model nuclear interaction plus a screened Coulomb potential, a model for NSM. The same model was already used in [8]. These simulations were done at the same temperature and densities, and under all three PBC geometries but for only one size. Results are presented and discussed in section 5 .

We close with some concluding remarks in section 6

\section{Molecular Dynamics model}

In this work we use a classical molecular dynamics model, $C M D$ [26], based on the work of V. Pandharipande. [27]. It has been very fruitful in nuclear studies of, among other phenomena, neck fragmentation [28], phase transitions [29], critical phenomena [30, 31], the caloric curve [32, 33], and isoscaling [34, 35] all without any adjustable parameters. Recently we extended it 
to be used in the study of NSM [8]. We include here a brief synopsis but readers are directed to these references for further details on the model.

$C M D$ treats nucleons as classical particles interacting through a two-body potential and solves the coupled equations of motion of the many-body system to obtain the time evolution of all particles. Since the $(\mathbf{r}, \mathbf{p})$ information is known for all particles at all times, it's possible to know the structure of the nuclear medium from a particle-wise perspective.

$C M D$ uses the phenomenological potentials developed by Pandharipande [27]:

$$
\begin{aligned}
V_{n p}(r) & =V_{r}\left[\exp \left(-\mu_{r} r\right) / r-\exp \left(-\mu_{r} r_{c}\right) / r_{c}\right] \\
& -V_{a}\left[\exp \left(-\mu_{a} r\right) / r-\exp \left(-\mu_{a} r_{a}\right) / r_{a}\right] \\
V_{n n}(r) & =V_{0}\left[\exp \left(-\mu_{0} r\right) / r-\exp \left(-\mu_{0} r_{c}\right) / r_{c}\right],
\end{aligned}
$$

where $V_{n p}$ is the potential between a neutron and a proton and it's attractive at large distances and repulsive at small ones, and $V_{n n}$ is the interaction between identical nucleons and it's purely repulsive. Notice that no bound state of identical nucleons can exist. It has many common features with potentials used by other models [6].

The cutoff radius is $r_{c}=5.4 \mathrm{fm}$ after which the potentials are set to zero. Calculations we present in this work were made with the Medium parametrization of the Pandharipande potentials. With this parametrization, symmetric infinite nuclear matter (NM) has an equilibrium density of $\rho_{0}=0.16 \mathrm{fm}^{-3}$, a binding energy $E\left(\rho_{0}\right)=16 \mathrm{MeV} /$ nucleon and a compressibility of about $250 \mathrm{MeV}$ [27].

As for the Coulomb interaction, we use a screened Coulomb potential of the form

$$
V_{C}^{(S c r)}(r)=\frac{e^{2}}{r} \exp (-r / \lambda)
$$

The correct approximation requires the parameter $\lambda$ to be the Thomas-Fermi screening length given by

$$
\lambda=\left[\frac{\hbar^{2}\left(96 \pi^{2}\left\langle\rho_{e}\right\rangle\right)^{1 / 3}}{m e^{2}}\right]^{1 / 2}
$$

as given in [2], where $m$ is the electron mass, and $\left\langle\rho_{e}\right\rangle$ is the electron gas number density, equal to that of the protons. The size of the simulation cell should be significantly larger than $\lambda$. Since the fulfillment of this requisite leads to prohibitively large systems, it has become a standard to artificially set $\lambda=10 \mathrm{fm}$ and use the first image convention to evaluate the forces [4, 6]. However, as we discuss in [7], for this model of nuclear interaction a value of $\lambda=20 \mathrm{fm}$ is needed to correctly produce the known pasta phenomenology.

The trajectories of individual nucleons are tracked using a Verlet algorithm and to control the temperature of the system we use an Andersen thermostat [36]. To achieve almost zero temperature, we set the thermostat to succesively lower temperatures by small steps letting the system reach thermal equilibrium at every step until $T=0.1 \mathrm{MeV}$ is reached. At that temperature, the nucleons are almost frozen, but we further cool it to $T=0.001 \mathrm{MeV}$ so that thermal fluctuations can be safely neglected. 


\section{Analitical results in different cell geometries}

\subsection{Cell geometries}

Periodic boundary conditions can be imposed on any polyhedra that fills space by translations. In order to fulfill that requirement, each face of the cell must have another face parallel to it and in the exactly opposite side of the cell, which earns this polyhedra the denomination of parallelohedra[37].

In this section we describe the three different unit cells we used in our simulations: cubic, hexagonal prism (HP) and the truncated octahedron (TO) or cubo-octahedron. To quantify the size of each cell we use the distance between opposing faces $L_{x}$, where $x$ is $C$ for cubic cell, $H P$ for hexagonal prism cell and $T O$ for the truncated octahedron. As a measure of the 'sphericity' of each cell, we calculate the ratio between $s_{x}$ the circumsphere radius and the inscribed sphere radius as in [37].

\subsubsection{Cube}

It's the most commonly used for its simplicity. The cubic cell and its images pack as a simple cubic lattice. It has side length $L_{c}, 6$ square faces of area $L_{c}^{2}$ and a volume of $V_{C}=L_{c}^{3}$. The total surface area of the cell is $S_{C}=6 L_{c}^{2}$ and the surface to volume ratio for a unit volume cell is $\frac{S_{C}}{V_{C}}=6$ The ratio between circumsphere and inscribed sphere radii is $s_{C}=\sqrt{3} \approx 1.73$.

\subsubsection{Hexagonal prism}

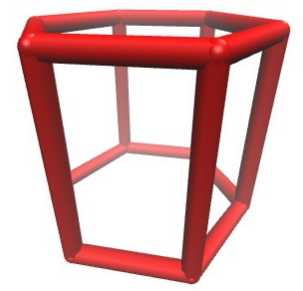

Figure 1: Hexagonal Prism

The HP cell has 6 rectangular faces and 2 hexagonal faces (see figure 2). It packs in an hexagonal honeycomb in the plane of the hexagonal faces and stacks evenly along the direction normal to that plane. The HP has a discrete rotational symmetry of order 6 about the axis normal to the hexagonal faces and this symmetry has an impact in the structures which is interesting to explore. We chose its dimensions so that every face is at the same distance $\left(L_{H P}\right)$ from its opposite face. With these proportions the prism has a height of $L_{H P}$ in the, say $z$ direction, normal to the hexagonal faces. The hexagonal faces have area $\frac{\sqrt{3}}{2} L_{H P}^{2}$ each. The lateral rectangular faces are $L_{H P}$ high and $\frac{\sqrt{3}}{3} L_{H P}$ (their surface area is, then $\frac{\sqrt{3}}{3} L_{H P}^{2}$ ).

The total surface area of the HP is $S_{H P}=2 \sqrt{3} L_{H P}^{2}$ and its volume $V_{H P}=\frac{\sqrt{3}}{2} L_{H P}^{3}$. The surface to volume ratio of the HP cell for unit volume is then $\frac{S_{H P}}{V_{H P}}=\frac{6}{L_{H P}}$, which for unit volume 
is $S_{H P}^{1} \approx 5.72$. The ratio between circumsphere and inscribed sphere radii for the HP is $s_{H P}=$ $\sqrt{\frac{7}{3}} \approx 1.53$.

\subsubsection{Truncated octahedron}

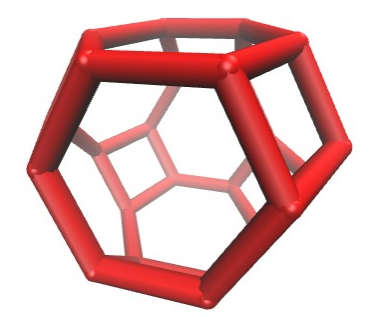

Figure 2: Truncated Octahedron

The TO is the Wigner-Seitz cell of the BCC Bravais lattice. As such, it packs as BCC. It has 8 hexagonal faces and 6 square faces. By construction, the distance between the center of the cell to any face is the same. A TO can be inscribed in a cube of side $L_{T O}$ and has exactly half its volume, then its volume is $V_{T O}=\frac{L_{T O}^{3}}{2}$. The surface area of each hexagonal face is $\frac{3 \sqrt{3} L_{T O}^{2}}{8}$ and for each square face it's $\frac{L_{T O}^{2}}{8}$. The TO cell's total surface area is $S_{T O}=\frac{3(1+2 \sqrt{3}) L_{T O}^{2}}{4}$. Its surface to volume ratio is $\frac{S_{T O}}{V_{T O}}=\frac{3(1+2 \sqrt{3})}{2^{\frac{4}{3}}} \approx 5.31$.

With a value of $s_{T O}=\sqrt{\frac{5}{3}} \approx 1.29$ the TO is the "most spherical" cell of the three.

To summarize, the cubic cell has the largest surface to volume ratio of the three cells, followed by the hexagonal prism and then the truncated octahedron. The cubic cell is also the farthest from spherical of the three. The HP, even if it is closer to spherical than the cube, is less isotropic than the other two. Among these three, the less biasing option seems to be the TO since it's the most spherical, the most isotropic and has the lowest surface to volume ratio. The only advantage of the cubic cell seem to be its computational economy.

\subsection{Surface area of traditional shapes in other geometries}

As we showed in [10], simulations of symmetric NM or Lennard-Jones-like systems at $\rho<\rho_{0}$, will produce non-homogeneous structures due to PBC at low enough temperature. When using a cubic cell, shapes are limited to one sphere, one cilindrical rod, one slab or their complementary shapes (cilindrical hole, spherical hole). Except for small transition regions, these shapes exhaust the possible solutions. We also showed that, at a given volume fraction, the stable configuration was that which had the least surface area among these five options. To compute the surface area for each shape we had explicitly assumed they were inscribed in a cubic cell under PBC. Because of the PBC, cilinders and slabs formally extend to other cells, thus the apparent surface of these structures which lies on the edges of the cells is not considered. 
In this section we extend those calculations, restricted to the same set of shapes, but now inscribed in each of the three periodic cells described in section 3 .

Simulations show that rods and slabs favor certain orientations related to the particular symmetries of each cell. For example, rods pierce the cell orthogonally to some pair of opposing faces, avoiding edges. Moreover, rods are always orthogonal to faces of the largest area possible (i.e. the hexagonal faces in both HP and TO, see section 3)

To compute the effective surface area of a rod we treat it as an open cilinder (without lids) of length $L$, the distance between faces, and set its radius based on the desired volume fraction.

As for slabs, the opposite is true: they are always parallel to the hexagonal faces in both HP and TO cells.

In the TO, a slab parallel to hexagonal faces cuts the cell through two of the square faces. Our calculation has implicitly the additional constraint that the slab is thinner than the side length of the square faces. Incidentally, that limiting case occurs at volume fraction $u=\frac{1}{2}$, where the slab actually becomes a 'slab-shaped hole'.

Table 1 shows the analytic expresions for the surface area of the usual simple shapes (sphere, rod, slab) for a given volume fraction $u$ and cell size $L$. The first column of table 1 contains the values of $L$ for a cell of unit volume of the corresponding geometry.

Table 1: Surface area for simple shapes under different PBC at volume fraction $u$ and cell of length $L_{x} . L_{x}^{1}$ is the value of the parameter $L_{x}$ for which a given cell has unit volume.

\begin{tabular}{c|c|c|c|c}
\hline \hline & $L_{x}^{1}$ & Sphere & Cilinder & Slab \\
\hline Cube & 1 & $(6 \sqrt{\pi} u)^{\frac{2}{3}} L_{C}^{2}$ & $\sqrt{4 \pi u} L_{C}^{2}$ & $2 L_{C}^{2}$ \\
HP & $\left(\frac{2 \sqrt{3}}{3}\right)^{\frac{1}{3}}$ & $3(\sqrt{\pi} u)^{\frac{2}{3}} L_{H P}^{2}$ & $\sqrt{\sqrt{12} \pi u} L_{H P}^{2}$ & $\sqrt{3} L_{H P}^{2}$ \\
TO & $2^{\frac{1}{3}}$ & $(3 \sqrt{\pi} u)^{\frac{2}{3}} L_{T O}^{2}$ & $\sqrt{2 \pi u} L_{T O}^{2}$ & $\sqrt{2} L_{T O}^{2}$ \\
\hline
\end{tabular}

We remind the reader that the line of argument is that in absence of Coulomb interaction, binding energy to first order can be divided in just a bulk plus a surface term. At $T=0$ the most stable structure is that which minimizes energy. Then, at a given volume fraction, energy minimization is achieved by surface minimization. In figure 3 we plot the least surface area (among those in table 1) as a function of volume fraction $u$ for the three cell geometries and unit volume cell (see caption for details).

The most evident feature of fig. 3 is that the regions of stability of each shape are different for each cell geometry. In particular, at any volume fraction between $\sqrt{3} /(2 \pi)$ and $8 \pi / 81$ (black vertical lines in fig. 3), the most stable shape would be a sphere in the TO cell, a rod in the cubic cell and a slab in the HP cell. This calculation predicts the same would happen for holes at the complementary volume fractions, but this is not exactly true in simulations because of the finite range of the potentials.

Qualitatively, it's reasonable that the TO being the "most spherical" cell should be able to lodge larger spheres or spherical holes than the others before the cell becomes too small to avoid interaction between replicas. This is clearly reflected in fig 3 .

But perhaps the most striking feature is that for the TO, the region at which rods are most stable is very small: between $8 \pi / 81 \approx 0.310$ and $1 / \pi \approx 0.318$. It's important to stress that the only physical assumption we made was that only bulk and surface energy contributions are 

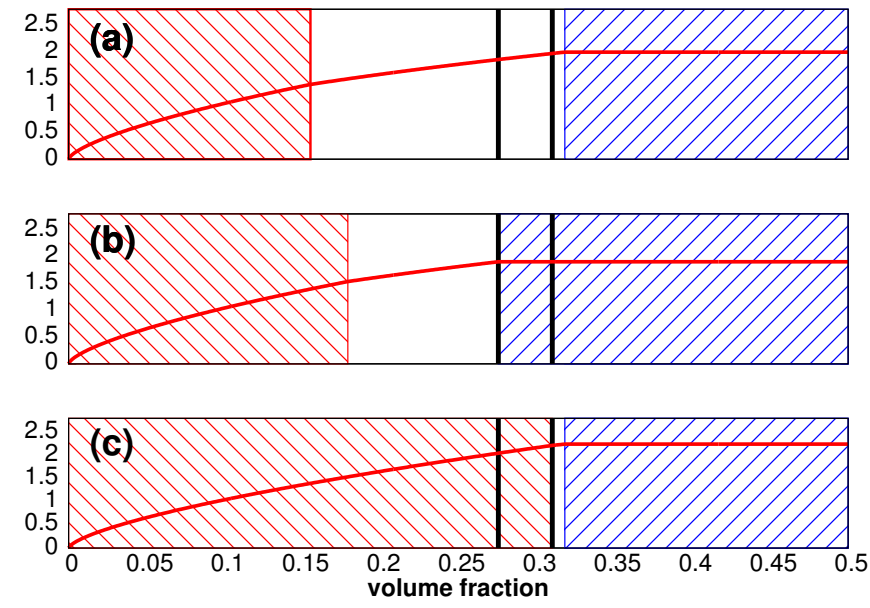

Figure 3: (Color Online) Least surface area among simple shapes under various PBC for unit volume. Panel $(a)$ for the cubic cell, $(b)$ for the HP cell and panel $(c)$ : for the TO cell. Upwards striped (red) areas indicate regions where spheres are the best solution, blank areas indicate regions where cilinders are, and finally, downward striped (blue) indicate regions where slabs are the best solution. In the region between the solid vertical black lines, the best solution is different in each cell.

relevant and PBC are imposed. Whenever that is true, (most notably when only LJ-likepotentials are involved) these results hold. And this is independent of the size of the cell since the surface area of every shape scales with $L^{2}$. One should be mindful about this fact when interpreting results from numerical simulations that rely on $\mathrm{PBC}$.

\section{Results of simulations without Coulomb interaction}

In the previous section we showed, by using simple geometric calculations, that for any system that has only bulk and surface contributions to energy under PBC, the particular geometry of the cell has a great impact on which shape has the least surface area. In those calculations we only considered the three traditional simple shapes: sphere, rod and slab. While those three shapes almost exhaust the possible results from simulations of large enough Lennard-Jones and NM systems [9, 10, 12] in cubic cells, it's not clear that these three shapes constitute a representative set of the possible results in other geometries.

To test the results from the previous section and to search for a set of possible shapes for PBC other than cubic, we performed molecular dynamics simulations using the three periodic cells described in section 3 . Since volume fraction is intimately related to number density, simulations were performed for three densities of interest. Furthermore, to explore how system size effects come into play in each geometry, simulations were made with both $A=1728$ and $A=4096$ particles.

In fig. 4 we show configurations at almost $T=0$ (see section 2 for $\rho=0.05 \mathrm{fm}^{-3}$ and both sizes. At this density there is no noticeable difference between the results in different cells. 


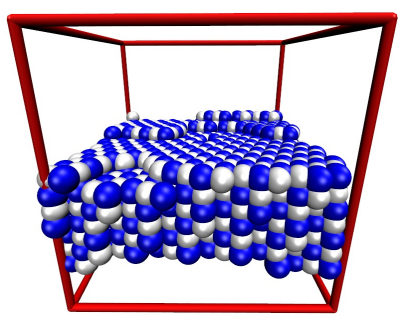

(a)

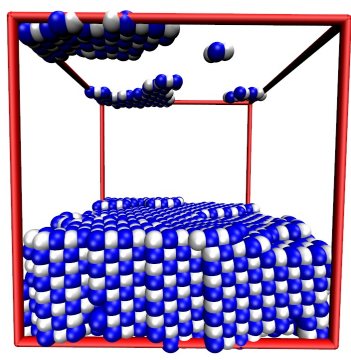

(d)

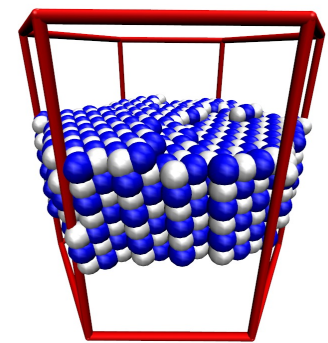

(b)

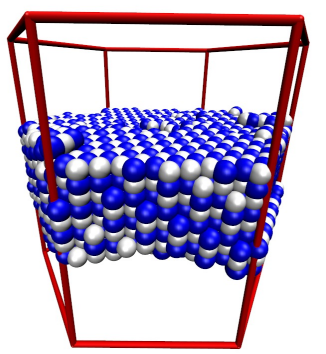

(e)

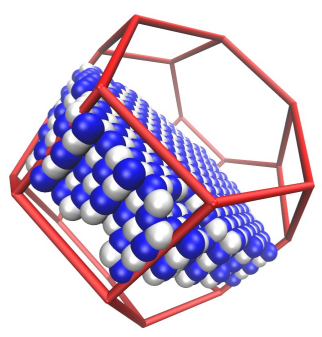

(c)

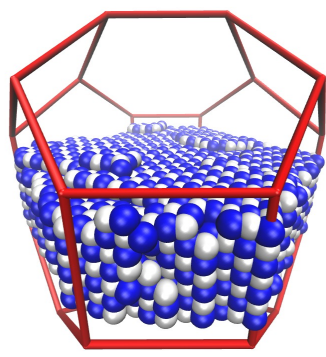

(f)

Figure 4: (Color online) Configurations at $T=0.001 \mathrm{MeV}$ for $A=1728$ (top row) and $A=4096$ (bottom row) nucleons at density $\rho=0.05 \mathrm{fm}^{-3}$ without Coulomb interaction. Panels $(a)$ and $(d)$ correspond to cubic cells, $(b)$ and $(e)$ to HP cells and panels $(c)$ and $(e)$ to TO cells.

In fig. 5 we present results at $\rho=0.08 \mathrm{fm}^{-3}$. At this density there is no qualitative difference between the shapes found with different system sizes at a given cell geometry, but each cell geometry yields a different result: Cilindrical holes for cubic, slabs for HP and spherical holes for TO.

In fig. 6 we present results at $\rho=0.10 \mathrm{fm}^{-3}$. Again, size has no qualitative effect on the shape that is stable for each geometry but that shape is different in each cell. For cubic and TO the stable shape is a spherical hole and for the HP it's a slab.

The results from this section clearly show that the particular (and a priori arbitrary) PBC conditions imposed on an MD simulation of cold nuclear matter (or any "bulk plus surface energy" system) can be determinant of the resulting configurations. And, while the shape may change, there is systematically only one structure per cell, independently of size and geometry of the cell. The fact that the shape of every solution is determined exclusively by surface minimization under the particular PBC means it is a solution to the Plateau's problem [23]. It then becomes obvious that complex non-homogeneous solutions (i.e. not spherical) must inherit a subset of the cell's symmetries. 


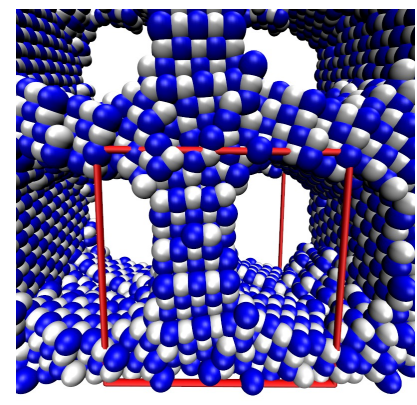

(a)

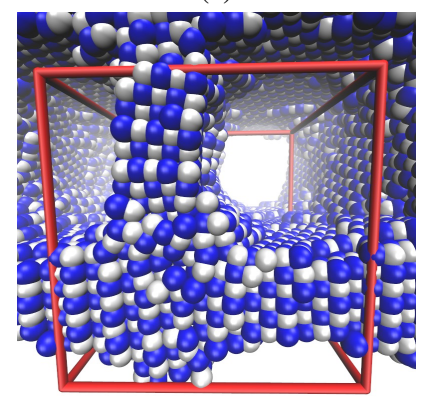

(d)

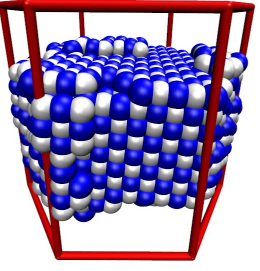

(b)

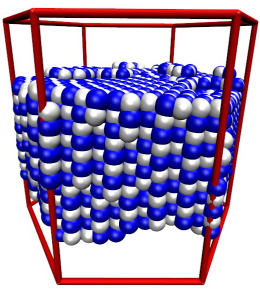

(e)

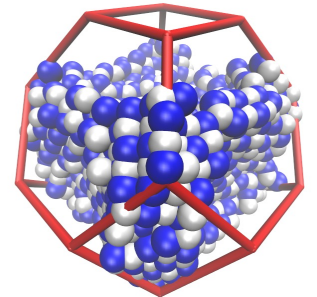

(c)

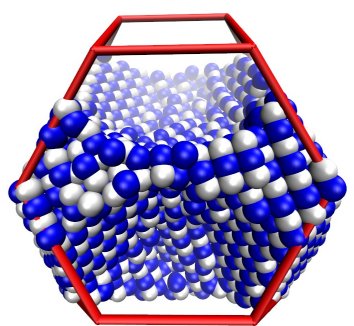

(f)

Figure 5: (Color online) Configurations at $T=0.001 \mathrm{MeV}$ for $A=1728$ (top row) and $A=4096$ (bottom row) nucleons at density $\rho=0.08 \mathrm{fm}^{-3}$ without Coulomb interaction. Panels $(a)$ and $(d)$ correspond to cubic cells, $(b)$ and $(e)$ to HP cells and panels $(c)$ and $(e)$ to TO cells.

\section{Results of simulations with Coulomb interaction}

In the previous section we analysed the effect of cell geometry on the possible solutions of NM simulations at sub-saturation densities under PBC. The results from MD simulations were consistent with the simple geometric calculations of section 3.2 Those calculations assumed that only bulk and surface terms were relevant and apply to every such system. In this section perform similar simulations but including a form of Coulomb interaction in the spirit of the Debye approximation (see 2), as a model of NSM. As stated before, the screening length must be set to, at least, $\lambda=20 \mathrm{fm}$ (a thorough analysis of this issue is presented in see [7]). Systems of $A=1728$ particles are too small for this purpose for amlost every density studied. And since working with larger systems in the non-cubic cells is computationally very expensive, we only studied systems of $A=4096$ particles in the HP and TO cells. In cubic cells simulations were performed also for $A=9826$ particles. For that size and the denisities studied, every cell is large enough.

In fig. 7. we show results for simulations at $\rho=0.05 \mathrm{fm}^{3}$ and for the three cell geometries (see figure caption for details) with Coulomb interaction. For every cell, "lasagna"-like structures are found, and more than one per cell, as expected.

However, the lasagna found in each cell is different from that of other cells. For example, in the cubic cell (fig7 (a)) there are lasagna of two and three particles wide, as in the TO, but in the 


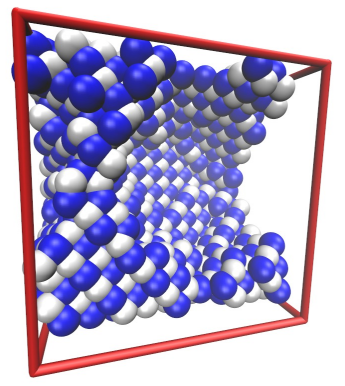

(a)

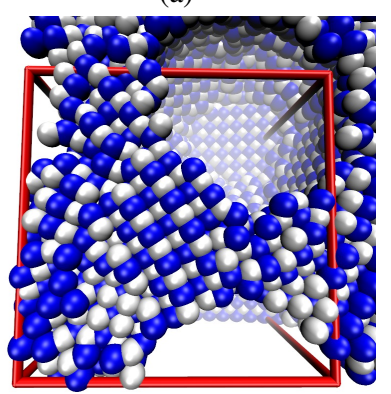

(d)

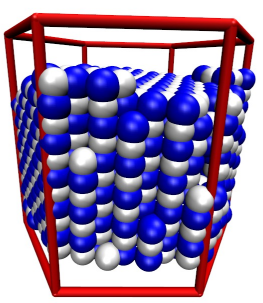

(b)

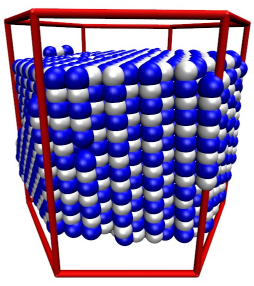

(e)

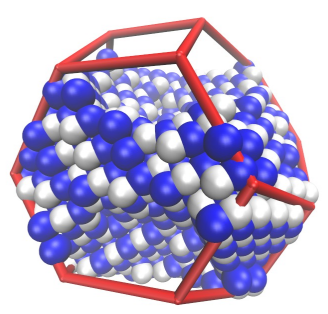

(c)

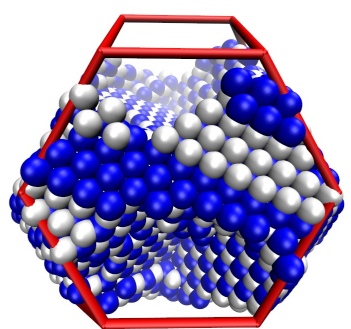

(f)

Figure 6: (Color online) Configurations at $T=0.001 \mathrm{MeV}$ for $A=1728$ (top row) and $A=4096$ (bottom row) nucleons at density $\rho=0.10 \mathrm{fm}^{-3}$ without Coulomb interaction. Panels $(a)$ and $(d)$ correspond to cubic cells, $(b)$ and $(e)$ to HP cells and panels $(c)$ and $(e)$ to TO cells.

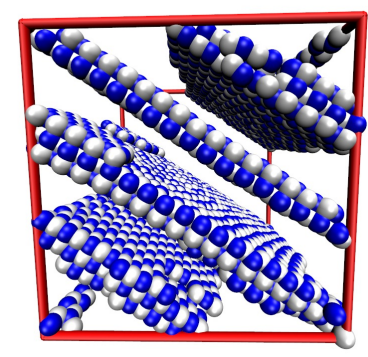

(a)

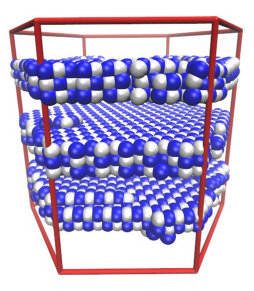

(b)

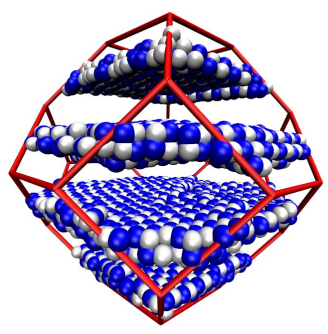

(c)

Figure 7: (Color online) Configurations at $T=0.001 \mathrm{MeV}$ for $A=4096$ nucleons at density $\rho=0.05 \mathrm{fm}^{3}$ with a screened Coulomb interaction in a cubic cell (panel $(a)$ ), HP cell (panel $(b)$ ) and TO cell (panel $(c))$.

HP every slab is three particles wide. Moreover, the distance between slabs is also different in 
each cell.

Under this circumstances it would be unwise to try to extract information about the length scale of density fluctuations without studying larger systems at the same conditions.

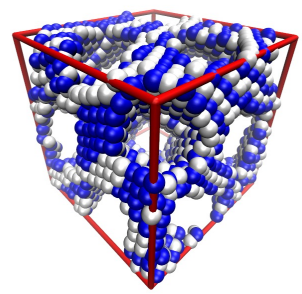

(a)

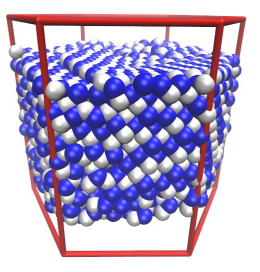

(b)

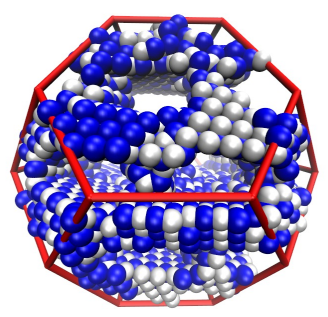

(c)

Figure 8: (Color online) Configurations at $T=0.001 \mathrm{MeV}$ for $A=4096$ nucleons at density $\rho=0.08 \mathrm{fm}^{3}$ with a screened Coulomb interaction. Panels $(a)$ corresponds to a cubic cell, $(b)$ to an HP cells and panel $(c)$ to the TO cell.

In fig. 8 we show results for simulations at $\rho=0.08 \mathrm{fm}^{3}$ for the three cell geometries (see figure caption for details). At this density, both cubic and TO cells consistently yield several cilindrical tubes per cell. In the HP cell, however, the solutions is a single slab.

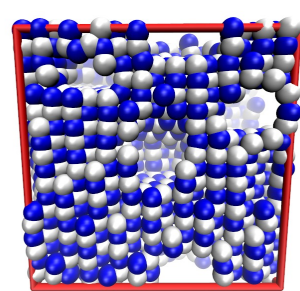

(a)

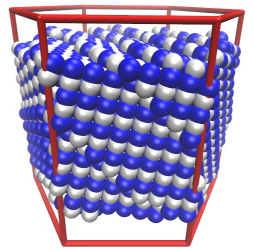

(b)

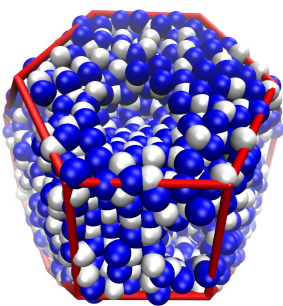

(c)

Figure 9: (Color online) Configurations at $T=0.001 \mathrm{MeV}$ for $A=4096$ nucleons at density $\rho=0.10 \mathrm{fm}^{3}$ with a screened Coulomb interaction. Panels $(a)$ corresponds to a cubic cell, $(b)$ to an HP cells and panel $(c)$ to the TO cell.

At density $\rho=0.10 \mathrm{fm}^{3}$ (shown in fig. 99 again the cubic and TO cell yield the same solution (in this case, several compact, unconnected holes with no apparent order). But the HP cell still yields a single slab.

It seems that in a HP cell of these proportions, for this system size and at relatively high densities, the mechanism responsible for the formation of non-homogeneous structures without Coulomb interaction (surface minimization exploiting the artificial PBC) is still present. It's 
present and is strong enough to overwhelm the disrupting effect of Coulomb interaction, which is enough to produce several structures in cells of the same volume but of different geometry. Thus we find that not only the size, but the geometry and symmetries of the cell may affect in unexpected ways the solutions of simulations even in systems with competing interactions.

Of course, these are indeed finite size effects which are likely to become less relevant for larger systems, we do not claim otherwise. What we find interesting noting is that finite size effects manifest in different ways in different cells, and that having more than one structure per cell is not necessarily enough to guarantee that they can be ignored. Specially when attempting to extract quantitative information on the scale of density fluctuations from the simulations.

These results suggest that for nuclear pasta simulations of this sizes, the actual length scale of density inhomogeneities is set as much by the interaction model as by the size and shape of the simulation cell.

For systems of $A=9826$ particles in cubic cells, the results are almost the same as for $A=4096$ particles. In particular, for $\rho=0.05 \mathrm{fm}^{3}$, the slabs now have a uniform width of three paricles

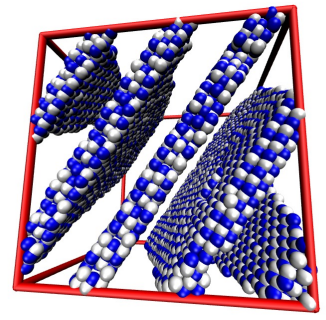

(a)

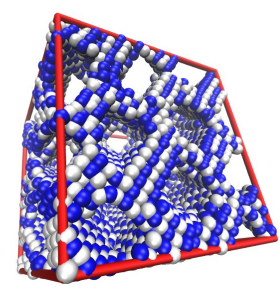

(b)

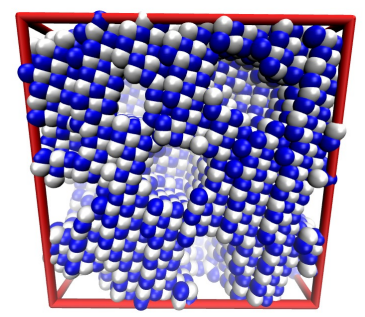

(c)

Figure 10: (Color online) Configurations at $T=0.001 \mathrm{MeV}$ for $A=9826$ nucleons with a screened Coulomb interaction at several densities in cubic cells. Panels $(a)$ corresponds $\rho=$ $0.05 \mathrm{fm}^{3},(b)$ to $\rho=0.08 \mathrm{fm}^{3}$ and panel $(c)$ to $\rho=0.10 \mathrm{fm}^{3}$.

\section{Concluding remarks}

In this work we show that simulations of symmetric NM using a semi-classical model produce, at subsaturation densities and low temperatures, non-homogeneous solutions reminiscent of the "nuclear pasta" expected in NSM but without a Coulomb interaction. The solutions are shaped as a spherical drop, a cilinder, a slab or their complements (cilindrical or spherical bubble) depending on the number density, but only one per cell. By considering only surface and bulk contributions to energy, we show that these non-homogeneous solutions are minimal surface configurations under PBC and, as such, are artificial. We explicitly prove this fact by showing that different primitive cell's geometries produce different solutions at the same physical conditions (i.e. density and temperature). The same is true for any system of particles interacting through Lennard-Jones-like potentials (i.e., short-ranged, attractive and with a hard core). Moreover, re- 
sults from [12] suggest that at sufficiently low but finite temperatures (namely, sub-critical), this results shoud approximately describe the behavior of the liquid phase in coexistence.

More importantly, we show that, even if this behavior is indeed a finite size effect, it is independent of the actual size of the system (if large enough) and it does not vanish for infinitely large systems.

The core mechanism behind these behavior is that at sub-saturation densities and low enough temperatures, particles tend to coalesce into a single, large, droplet. But as the droplet grows and approaches the size of the cell, it begins to interact with its own replicas in neighboring cells through the artificial PBC. Then, the PBC allows the system to lower its energy by trading wouldbe surface for bulk, forming the pseudo-pasta. The system minimizes its energy by adopting a minimal surface configuration, but the configuration that minimizes the surface at a given volume fraction depends on the cell's geometry and has no physical meaning.

In other words, for LJ-like systems, the only length scale, as well as the symmetries of the solution at low temperatures, are set exclusively by the simulation cell. And so, inhomogeneities are artificial.

On the other hand, if a screened Coulomb interaction is included, in addition to the LJlike potential, a new, physically meaningful length scale appears. This length scale is related to the maximum size an isolated droplet may have before the disruptive effect of the repulsive interaction breaks it down into smaller droplets. The same is true for any system of particles with competing interactions of different ranges. However, even in this case, the artificial PBC imposed can bias or constraint the length scale of the inhomogeneities, or even impose symmetries, if the system is not large enough. Our simulations show that this is the case for systems not too large, since for some physical conditions the result depend on the cell used. In particular, for $\rho=0.08 \mathrm{fm}^{-3}$, the cubic and TO cells produce several cilindrical tunnels, yet for the same physical conditions, the HP cell yields a single slab.

In NSM simulations, it is acceped that a simulation or model that yields a single structure per cell suffers from finite size effects. This result show that producing multiple structures per cell is, by no means, evidence enough that the solution is free of such effects.

Nevertheless, it seems almost certain that for systems with competing interactions of different ranges, the artificial effects discussed in this work will become negligible if the system is large enough, as compared to the characteristic length scale set by the interactions. Only in this case the inhomogeneities observed will be due to only the interaction potentials and the physical conditions.

\section{acknowledgments}

C.O.D. is a member of the "Carrera del Investigador" CONICET supported by CONICET through grant PIP5969.

\section{References}

[1] D. G. Ravenhall, C. J. Pethick and J. R. Wilson, Phys. Rev. Lett. 27, 2066 (1983).

[2] R. D. Williams and S. E. Koonin, Nucl. Phys. A435, 844 (1985).

[3] A. L. Fetter and J. D. Wallecka, Quantum Theory of Many-Particle Systems, McGraw-Hill (1971)

[4] T. Maruyama, K. Niita, K. Oyamatsu, T. Maruyama, S. Chiba and A. Iwamoto, Phys. Rev. C57, 655 (1998)

[5] G. Watanabe, K. Sato, K. Yasuoka and T. Ebisuzaki, Phys. Rev. C66, 012801 (2002).

[6] C.J. Horowitz, M.A. Perez-Garcia, and J. Piekarewicz, Phys. Rev. C69, 045804 (2004)

[7] P.N. Alcain, P.A. Giménez Molinelli, J.I. Nichols and C. O. Dorso, arXiv:1311.5923 [nucl-th]. 
[8] C. O. Dorso, J. A. López, P. A. Giménez Molinelli, Phys. Rev. C86, 055805 (2012).

[9] K. Binder, B. J. Block, P. Virnau, A. Tröster, Am. J. Phys. 80129 (2012)

[10] P.A. Giménez Molinelli, J.I. Nichols, J.A. López and C.O. Dorso, Nucl. Phys. A 923 31-50 (2014)

[11] M. Schrader, P. Virnau and K. Binder, Phys. Rev. E 79061104 (2009)

[12] L.G. MacDowell, V.K. Shen and J.R. Errington, Jour. Chem. Phys. 125034705 (2006)

[13] D. Frenkel and B. Smit, "Understanding Molecular Simulations", 2nd Ed.,Academic Press (2002).

[14] G. Watanabe. K. Sato, L. Yasuoka amd T. Ebisuzaki, Phys. Rev. C69, 055805 (2004)

[15] C.J. Horowitz, M.A. Perez-Garcia, D.K. Berry and J. Piekarewicz, Phys. Rev. C72, 035801 (2005).

[16] W. G. Newton and J. R. Stone, Phys. Rev. C79, 055801

[17] K. Nakasato, K. Oyamatsu and S. Yamada, Phys. Rev. Lett. 103, 132501 (2009)

[18] S. Föster and T. Planterberg, Angew. Chem. Int. Ed. 41, 688 - 714 (2002)

[19] H. Sonoda, G. Watanabe, K. Sato, K. Yasuoka and T. Ebisuzaki, Phys. Rev. C77, 035806

[20] C. Ortiz, J. Lorenzana, C. Di Castro, Phys. Rev. Lett. 100, 246402

[21] J. Archer, C. Ionescu and L. Reatto, J. Phys. Conden. Matter 20, 415106 (2008)

[22] K. Larsson and F. Tiberg, Current Opinion in Colloid \& Interface Science 9 365-369 (2005)

[23] W. Gòżdz̀ and R. Holyst, Macromolecular Theory and Simulations, Vol. 5, 321-332 (1996)

[24] H. Pais and J. R. Stone, Phys. Rev. Lett. 109, 151101 (2012)

[25] E. A. Lord and A. L. MacKay, Current Science, vol. 85 No. 3, 10 August (2003)

[26] A. Barrañón, C.O. Dorso, J.A. López and J. Morales, Rev. Mex. Fís. 45, 110 (1999).

[27] A. Vicentini, G. Jacucci and V. R. Pandharipande, Phys. Rev. C31, 1783 (1985); R. J. Lenk and V. R. Pandharipande, Phys. Rev. C34, 177 (1986); R.J. Lenk, T.J. Schlagel and V. R. Pandharipande, Phys. Rev.

[28] A. Chernomoretz, L. Gingras, Y. Larochelle, L. Beaulieu, R. Roy, C. St-Pierre and C. O. Dorso, Phys. Rev. C65, $054613(2002)$

[29] A. Barrañón, C.O. Dorso, and J.A. López, Nuclear Phys. A791, 222 (2007).

[30] A. Barrañón, R. Cárdenas, C.O. Dorso, and J.A. López, Heavy Ion Phys. 17, 1, 41 (2003).

[31] C.O. Dorso and J.A. López, Phys. Rev. C64, 027602 (2001).

[32] A. Chernomoretz, C.O. Dorso and J.A. López, Phys. Rev. C64, 044605 (2001)

[33] A. Barrañón, J. Escamilla Roa and J.A. López, Phys. Rev. C69, 014601 (2004).

[34] C.O. Dorso, C.R. Escudero, M. Ison and J.A. López, Phys. Rev. C73, 044601 (2006).

[35] C.O. Dorso, P.A. Giménez Molinelli and J.A. López, J. Phys. G: Nucl. Part. Phys. 38115101 (2011); ibid, Rev. Mex. Phys., S 57 (1), 14 (2011).

[36] H.C. Andersen, J. Chem. Phys. 722384 (1980).

[37] D. Adams, CCP5 Information Quarterly, 10, 30-36 (1983) 